\title{
In House Enhanced 3D Printing of Complex AAA for EVAR Treatment Planning and Preoperative Simulation
}

\section{Hoffman Aaron*, Nitecki Samy, Engel Ahuva, Karram Tony, Leiderman Maxim Kogan Igor, Si-On Erez and Ofer Amos}

Department of vascular surgery and Transplantation and Department of Radiology, Rambam Health Care Campus, and Rappaport Faculty of Medicine, Technion, Haifa, Israel

\begin{abstract}
Three dimensional printing (3D printing) is becoming a common useful technology in many areas in medicine. This technology allows better appreciation of complex anatomical and pathologic conditions. In vascular medicine and surgery 3d modeling may become of help in Endovascular Aortic Repair (EVAR) treatment planning, especially in cases of complex angulations and branching at the aneurysm neck.

Accurate and rapid solid $3 \mathrm{~d}$ models were prepared in house using common and freely available software programs, and an accurate 3D printer. These solid models allowed palpation and manual handling and visual inspection for a better appreciation of complex AAA necks. Color enhancement of these models added another dimension of comprehension, even for experienced surgeons and invasive radiologists. A full sized model may allow more accurate measurements of branch distances and angles in space when tortuosity is severe. Fenestrations and "Chimney" branches may be easily envisioned preoperatively.

In addition, 3D hollow outer shells were constructed to obtain a workable AAA arterial wall-like model for rehearsal and preoperative stent graft deployment simulation. Various commercial stent grafts from several manufacturers were deployed under fluoroscopy to evaluate feasibility of the planned procedure. In simple AAA cases device deployment simulation was always successful, but in one AAA case with a severely angulated neck all devices failed.

We conclude that 3D printing and modeling and outer shell fabrication for preoperative simulation are helpful in EVAR planning of complex AAA.
\end{abstract}

Keywords: 3D printing; 3D model; AAA; EVAR; Stent graft; Chimney; Simulation

\section{Background}

Planning of treatment for patients with Abdominal Aortic Aneurysms (AAA) requires detailed preoperative knowledge of anatomical details, especially if Endovascular Aneurysm Repair (EVAR) is contemplated. The surgeon needs to measure accurately various diameters, lengths and angles, in order to choose the most suitable device from a large possible inventory of EVAR device manufacturing companies, sizes, limb diameters, flexibility, and more technical considerations. The length of the "Neck" of the aneurysm, the origin of visceral branches of the Aorta must be carefully considered to avoid inadvertent occlusion of vital branches, or to plan fenestrations or additional branch stents like "chimneys" that will protect a viscus from ischemia. A mistake in preoperative evaluation may lead to angular misalignment of the stent graft with the aortic wall, insufficient length of overlap, endoleaks, migration, limb occlusions, ruptures, or visceral malperfusion [1].

The conventional way to perform accurate preoperative measurements and stent planning is based on two dimensional (2D) reconstructions of the Computerized Tomography Angiography (CTA) images, and centerline based dimension measurements, or better so by three dimensional (3D) reconstructions of the 2D images. Dedicated software packages are now available for automatic recognition and naming of each artery and branch and for stent planning accordingly. These methods proved to be accurate and they allow the surgeon and the invasive radiologists excellent precision of planning in most AAA cases. However, in some cases of complex AAA, especially with short necks, severe juxta renal tortuosity and angulation, and proximity of visceral branches, the real dimensions are very difficult to comprehend even by experienced surgeons. It was felt that for those selected difficult cases better methods should be explored.

Additive manufacturing or rapid $3 \mathrm{D}$ printing is a method to manufacture a desired physical 3D model from a virtual image or from computerized planning software. Many 3D printers from various companies are commercially available now and they are rapidly becoming more friendly and easy to use. Some printers are even marketed as personal 3D printers. The various 3D printing machines available now have a wide range of capabilities, build materials, sizes, and prices. They have recently been used to improve diagnosis and treatment in various areas of medicine and surgery [2]. This technology allows better appreciation of complex 3D anatomic and pathologic conditions. In vascular medicine and surgery $3 \mathrm{~d}$ modeling may be of help in understanding the underlying pathology. In fact, actual 3D models of aneurysms have been industrially produced from CTA images for the purpose of stent design and to train physicians and other personnel by institutes and by EVAR manufacturing companies $[3,4]$.

Therefore, we have embarked on in-house rapid 3D printing

*Corresponding author: Aaron Hoffman, Director, Department of Vascular Surgery and Transplantation Rambam Medical Center, Haifa, Israel, Tel: 972-47772976; Fax: 972-4-7772976; E-mail: ahofman@rambam.health.gov.il

Received September 03, 2014; Accepted October 16, 2014; Published October 23, 2014

Citation: Hoffman A, Nitecki S, Engel A, Karram T, Leiderman M, et al. (2014) In House Enhanced 3D Printing of Complex AAA for EVAR Treatment Planning and Preoperative Simulation. J Cardiovasc Dis Diagn 2: 181. doi:10.4172/2329 9517.1000181

Copyright: ( 2014 Aaron $\mathrm{H}$, et al. This is an open-access article distributed under the terms of the Creative Commons Attribution License, which permits unrestricted use, distribution, and reproduction in any medium, provided the original author and source are credited. 
technology, in order to improve EVAR treatment planning, particularly in patients with complex AAA disease, when conventional virtual 3D modeling is not satisfactory.

\section{Materials and Methods}

\section{Personal computer processing}

All computerized 3D preparative work has been done on a departmental Personal Computer (PC). There is no need any more for a dedicated advanced work station to perform the needed calculations. AAA patients two Dimensional (2D) Computed Tomography Angiography (CTA) axial images, in Digital Image Communications (DICOM) format, were first viewed on any hospital based CT viewer that has access to the institutional PACS (picture archiving and communication system). A specific series and an area of interest that includes the segment of aorta above the aneurysm, where a possible "Landing zone" for a stent graft is anticipated, down to the femoral vessels was noted. Selection of the numbers of the slices, from the entire CT series, required for future reconstruction is determined.

Vessel analysis, segmentation, and surface rendering reconstructions, to prepare $3 \mathrm{~d}$ models can be done using commercial or free public medical imaging software packages like InVesalius ( $h t t p: / /$ svn.softwarepublico.gov.br/trac/invesalius). In this study we preferred to use the web based Philips Intellispace Portal package, available in our institute. Sub selection of the images that include the supraceliac to common femoral region, and then Advanced Vessel Analysis (AVA) surface rendering was used to prepare the $3 \mathrm{D}$ reconstruction. Bone was removed taking care not to remove vascular structures. Only a short length of visceral and internal iliac arteries was kept. Lumbar arterial branches were removed. Care was taken to keep accessory renal arteries and internal iliac arteries. Calcifications on arteries were kept if they did not distort the lumen. The final image (Figure 1) has been saved as a Standard Tessellation Language (STL) file format inside the portal and then exported to the PC. The typical preparation time of a printable $3 \mathrm{~d}$ stl file is only $15-20$ minutes long. The ensuing file size is usually less ten 1 megabyte. The completed .stl file can be viewed using any of a number of free $3 \mathrm{~d}$ viewers available on the web (Appendix 1).

\section{Considerations regarding size of the aneurysm}

The size of the ensuing 3D model is of consideration because of printer dimension limitations. All printers have a predefined tray size, with a maximal length usually of $10-25 \mathrm{~cm}$. If the patient's AAA is very large the $3 \mathrm{D}$ model may turn up to be too large or long for the specific printer. The prepared 3D model can then artificially be reduced in size to fit the limiting dimensions of the printer's tray. In this case the $3 \mathrm{D}$ product is effective for general impression and evaluation only but not for accurate measurements (Figure 1). Another solution to the size limit problem is to create the desired 3D model from 2 or more different $3 \mathrm{D}$ parts. This is done by sub selecting sets of images for separate complimentary $3 \mathrm{D}$ reconstructions and printed so that the anticipated final model will be assembled from 2 or more physical parts that can be glued together later (Figure 2). Another option is to $3 \mathrm{D}$ print only the area considered to be most problematic, i.e. the juxtarenal region of the Aorta and the aneurysm. In this case the neck and visceral branches can be accurately evaluated but length measurements are not possible.

\section{Choice of a 3D printer}

Many commercial 3d printers are becoming available on the marketplace for personal use, with a wide range of capabilities, dimensions, build materials, and prices. The printer available in our medical center was Objet30 Pro. This 3D printer is almost desktop size and is conveniently located inside the faculty campus and used regularly by other groups for research and nonmedical purposes. The build materials that the printer can use include a wide range of various substances with different features. To produce a solid and accurate $3 \mathrm{D}$ AAA model we chose to use a polypropylene like material.

The previously prepared model in .stl file format is transferred to the printer operator personally, by email or by other media. The 3D model is loaded onto the printer's software, put on its virtual tray, and checked for printability. The apparatus software automatically converts the 3D model into very thin slices and plans build material and support material. The software optimizes position on the tray and calculates the amounts of materials to be used and the time of production. When given the order to print it starts adding printed slices layer by layer from the bottom up. Printing time depends on model size. A full sized model of a large AAA may take 24 hours or more to print. A reduced size model, a partial model, or a hollow model may print in only one hour.

\section{Enhancing 3D models with colors}

The basic color of the 3D model is white. This suits experienced

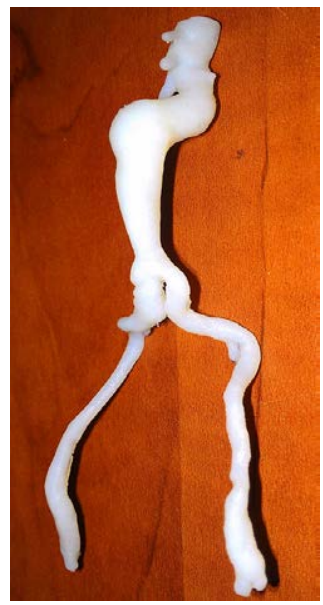

Figure 1: A reduce size 3D model of AAA.

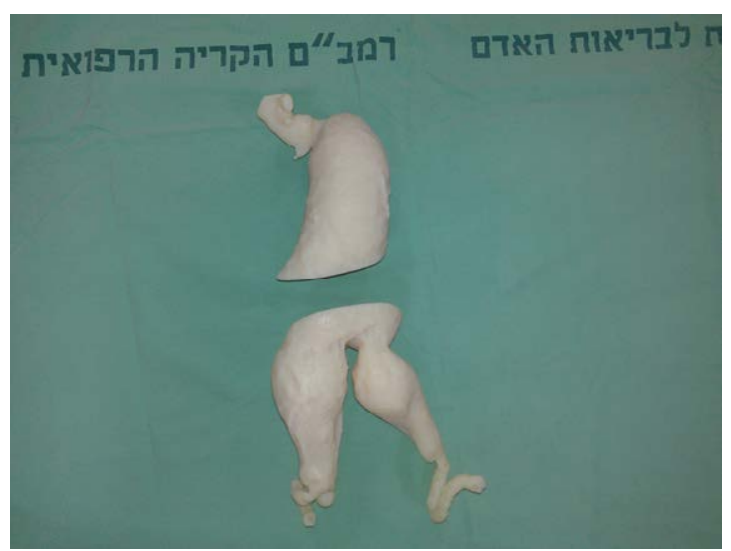

Figure 2: Two model parts of a large AAA. The parts are complimentary and will be glued together to form a single physical 3D model. 
Citation: Hoffman A, Nitecki S, Engel A, Karram T, Leiderman M, et al. (2014) In House Enhanced 3D Printing of Complex AAA for EVAR Treatment Planning and Preoperative Simulation. J Cardiovasc Dis Diagn 2: 181. doi:10.4172/2329-9517.1000181

surgeons and invasive radiologists who are used to watch white computer reconstructions of AAA in their daily work and to virtually rotate and manipulate them on the computer screen. However, we noted that trainees and less experienced personnel get a better understanding of the details of the aneurysm if the model is differentially painted for normal and abnormal findings. We chose to paint the normal part of the artery in red and the aneurysmatic parts in yellow (Figures 3 and 4).

\section{Production of a 3D hollow Shell}

For the purpose of preoperative rehearsal of EVAR, and for the purpose of training of personnel in endovascular techniques, a real sized phantom of the actual AAA arterial wall is desirable. This can be achieved by fabricating an outer hollow shell of the $3 \mathrm{D}$ model that closely resembles the real aortic wall [5]. We used various methods and materials to build this outer shell.

\section{Computer printed outer shell}

Several professional and free software programs are available for processing an outer shell for 3D printing. For this purpose we used the open source free version of Meshlab software (Version 1.3.2, Meshlab. com). This software is readily downloaded from the internet to the PC. The basic 3D model is imported to Meshlab and manipulated to create a $1.5 \mathrm{~mm}$ thick outer shell of the model (Appendix 2).

Printing of this thin outer shell is fast and much less material is used in this process, however the completed hollow model needs additional manipulations. Cutting out about $1.5 \mathrm{~mm}$ of the edges manually and removal of support material is needed to get a workable aortic wall like structure. The ensuing model is light but stable, and very accurate. Another advantage is that it is semi-transparent and branches are easily ready for guide wire cannulation (Figure 5).

However, the shell made of this material is stiff, unlike real arterial wall. This may interfere with the maneuverability of a stent graft device, and to falsely preclude the feasibility of a planned EVAR procedure in a complex AAA patient (Figure 6).

\section{Hollow shells of AAA made of silicone rubber}

So far no flexible and compliant material is available for the present 3D printer to produce an adequate outer shell. Therefore, handmade flexible shells have been prepared and tested. Different household type

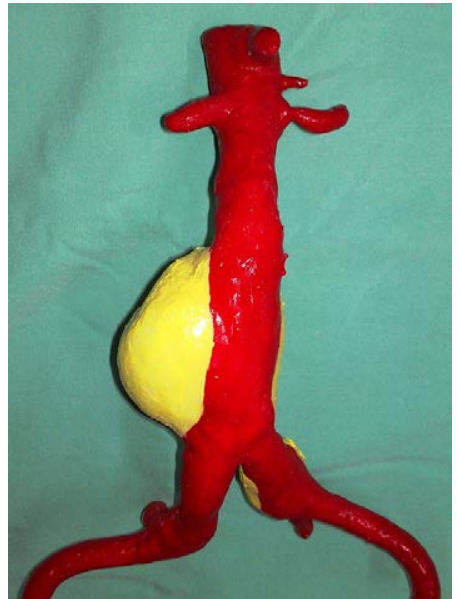

Figure 3: Color enhanced 3D model of AAA. "Normal" artery in red. Aneurysmatic parts in yellow.

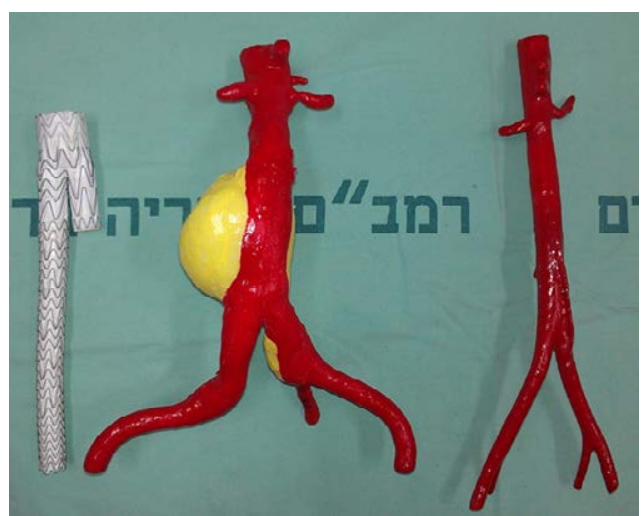

Figure 4: 3D models in EVAR planning. The AAA model in the middle, a stent graft on the left. A normal aorta 3D model on the right for comparison.

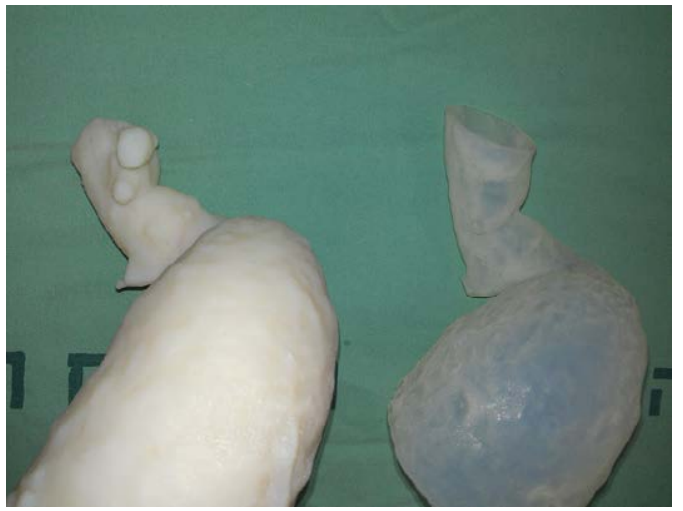

Figure 5: A solid 3D model of a complex AAA on the left and the corresponding outer shell on the right.

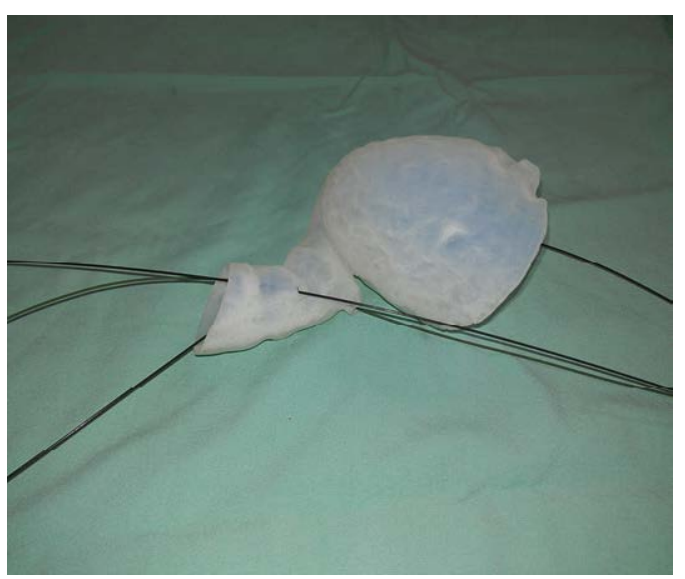

Figure 6: Guide wires inside the shell. One passes through the whole aneurysm, one through the SMA and one through the RT Renal artery.

silicone rubber gel was used. Firstly, a thick layer of Clear Silicone for household use or white Silicone RTV were put and sculptured onto the solid 3D model and allowed to dry for 24 hours. This outer shell is then sharply cut longitudinally, expanded manually, and the inner solid model is extracted. The longitudinal cut is closed by silicone glue (Figures 7 and 8). This method produces a real life size, flexible, hollow 
Citation: Hoffman A, Nitecki S, Engel A, Karram T, Leiderman M, et al. (2014) In House Enhanced 3D Printing of Complex AAA for EVAR Treatment Planning and Preoperative Simulation. J Cardiovasc Dis Diagn 2: 181. doi:10.4172/2329-9517.1000181

Page 4 of 7

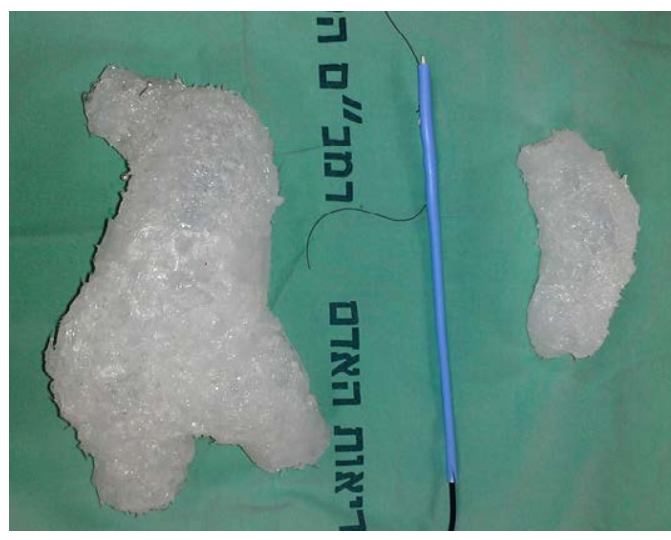

Figure 7: Clear silicone shells of 2 different AAA. The device in the middle contains the compressed stent graft for simulation.

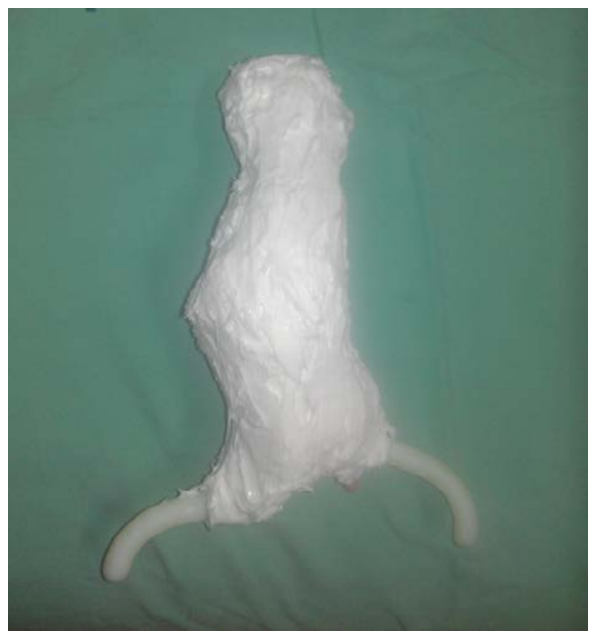

Figure 8: A white silicone shell around a 3D AAA model. The silicone will be cut longitudinally and the solid part extracted.

outer shell of the AAA, resembling in consistency a real arterial wall, and making it adequate for EVAR simulation.

\section{Fast drying impression materials}

These materials are frequently used by dentists and were made available to us from the dentistry department. We used Vinilpolysiloxane Elite Hydrorize fast set to create an outer shell. The two components of the material are poured on the 3D solid model and quickly sculptured around to create a 3-5 $\mathrm{mm}$ thick adherent layer. After a few minutes of drying the shell is sharply cut longitudinally and the solid 3D model extracted. The longitudinal cut is closed by the same material. A hollow 3D shell, resembling real aortic wall, is received. This material is partially flexible and allows near real life stent deployment (Figure 9).

\section{Fluoroscopy of the 3D solid models and of the shells}

We tested the solid 3D models and the various outer shells under fluoroscopy in the angiography suite, and proved that they are partially radiolucent. The different build materials of the outer shells that we tested did not interfere with the fluoroscopic visualization of the original aneurysmal sac of the specific patient. By remeasuring the fluoroscopic images of the various models we could also validate that the diameters and lengths of the $3 \mathrm{D}$ models, as well as their respective outer shells, precisely reproduced the actual dimensions of the original AAA's. Therefore these shells are deemed to be suitable for preoperative practice simulation in the angiography suite (Figure 10).

\section{Results}

The in house produced 3D solid models are smooth and accurate to the millimeter range. The tactile and visual inspection of a complex AAA model is helpful in better comprehending all minute details of the disease to improve treatment plans. The models we produced added significantly to all watchers including experienced EVAR surgeons, invasive radiologists, and product specialist of the EVAR device manufacturer.

Many aspects of the preoperative evaluation of a complex AAA may change due to new observations on a solid model. Some of these aspects are detailed as follows.

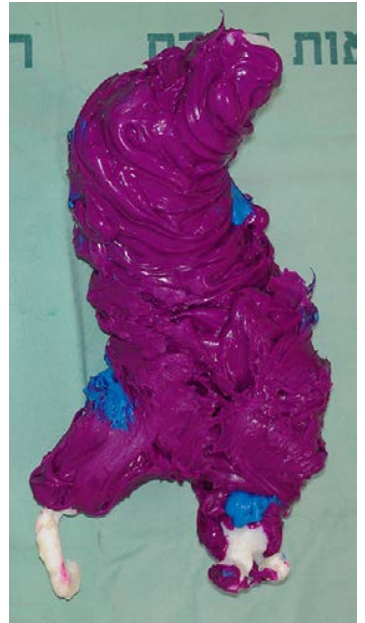

Figure 9: A shell made of fast set impression material (Hydrorize) around a 3D model.

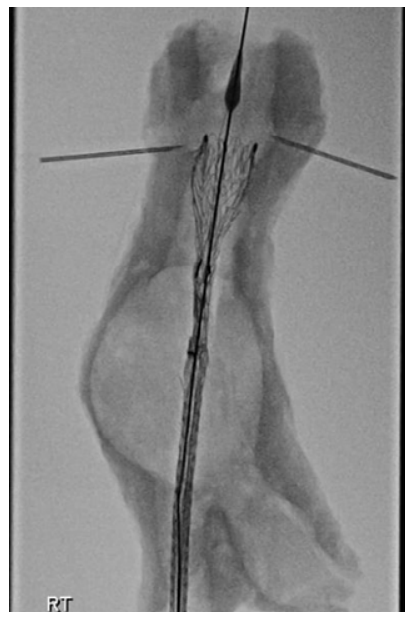

Figure 10: Fluoroscopy of stent graft inside a silicone shell (Gore device, partially deployed). The lumen of the aneurysm is clearly seen. The renal arteries are enhanced by radio opaque needles. 


\section{Details of aneurysm neck}

Neck length is of utmost importance for the primary success of any EVAR procedure. FDA approved Indications for the various devices require a minimum length of $15 \mathrm{~mm}$ of suitable aortic wall for tight overlap with the graft and for an efficient seal to avoid Type 1 endoleak. The mechanical features of the different devices also dictate maximal possible angles amenable for stent graft deployment. These prerequisite conditions are not always met, and in real life many procedures are performed off label. In some complex AAA cases the aneurysm neck is sharply angulated and tortuous in space so that the true dimensions cannot be precisely elucidated from the computer screen images, even after meticulous spatial manipulations and centerline corrections. The solid 3D models allow better measurements of these cases (Figure 11).

In one case we could demonstrate that the distance to the lower renal artery was actually greater than previously appreciated, and really could enable a safe infrarenal placement of the covered edge of the stent (Figure 12).

\section{Details of aortic branches}

Accurate details of important aortic branches can be visualized easily and their exact spatial origins from the Aorta, the presence and location of accessory renal arteries, the degree of stenoses, the severity of tortuosity and the distances to bifurcations, are better appreciated (Figure 13).

\section{Neck angulation}

The actual position that the stent graft will take inside the aortic sac after deployment is difficult to apprehend beforehand. In most AAA cases it is assumed that the relative rigidity of the metallic mesh of the stent will follow the centerline. However, especially in very large sacs and angulated necks the final position of the device is impossible to predict. This problem can be better understood when observing the variability of possible real centerlines of a 3D model (Figures 14 and $15)$.

\section{Simulation of stent graft deployment}

EVAR simulation was performed under fluoroscopy in the angiography suite with the Gore Excluder, and with the Valiant Thoracic devices. Simulations have been performed inside the outer shells mimicking the aortic wall of the aneurysms. Similar to the procedure in patients, a guide wire was first passed from the iliac artery orifice of the shell, across the aneurysmal sac and out of the upper

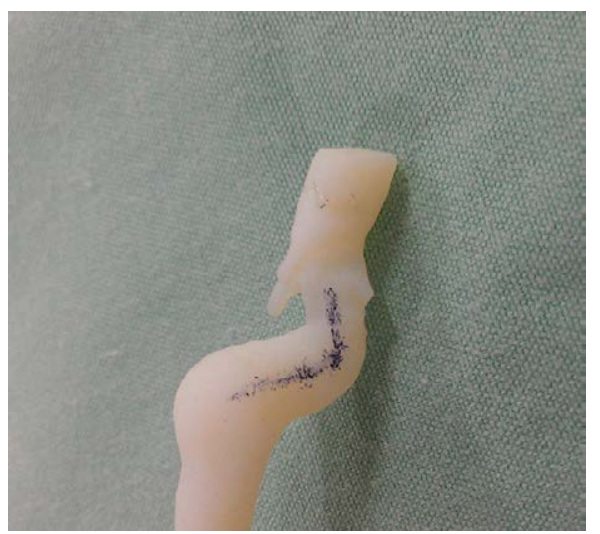

Figure 11: A 3D model to show fine details of the neck of the aneurysm.

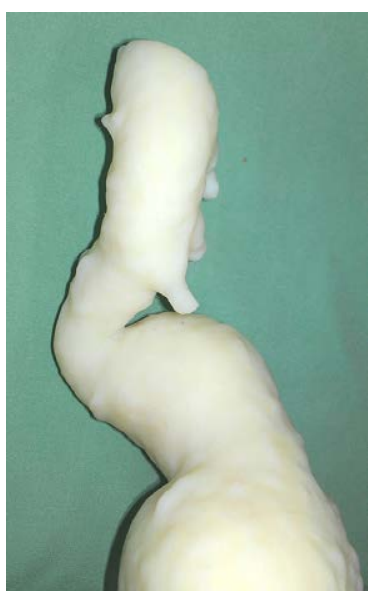

Figure 12: $A$ 3D model showing how the complex structure of the AAA hides the real length of the neck.

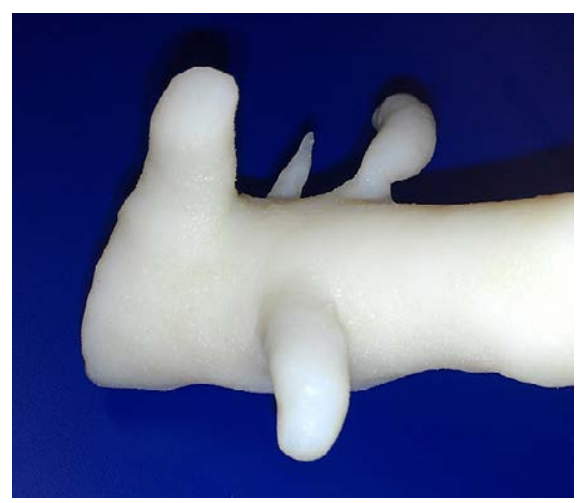

Figure 13: Size enhanced 3D model that shows on accessory Lt renal artery and a stenosis of the Lt main renal artery.

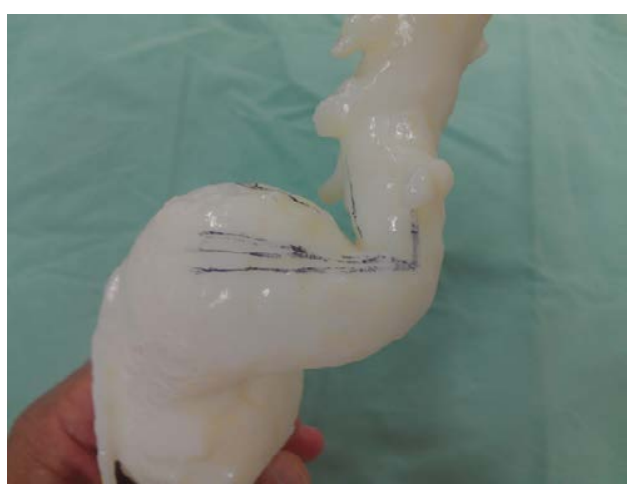

Figure 14: $A 3 D$ model of an $A A A$ with mild variation of possible centerlines and angles.

aortic orifice. This guide was then substituted with a stiff wire. The stent graft device was then delivered upon the stiff wire, pushed past the planned landing zone, partially opened and pulled back to the final desired position distal to renal orifices, and then completely deployed (Figures 16 and 17).

In one patient with a complex AAA, severe angulation, a short 
Citation: Hoffman A, Nitecki S, Engel A, Karram T, Leiderman M, et al. (2014) In House Enhanced 3D Printing of Complex AAA for EVAR Treatment Planning and Preoperative Simulation. J Cardiovasc Dis Diagn 2: 181. doi:10.4172/2329-9517.1000181

Page 6 of 7

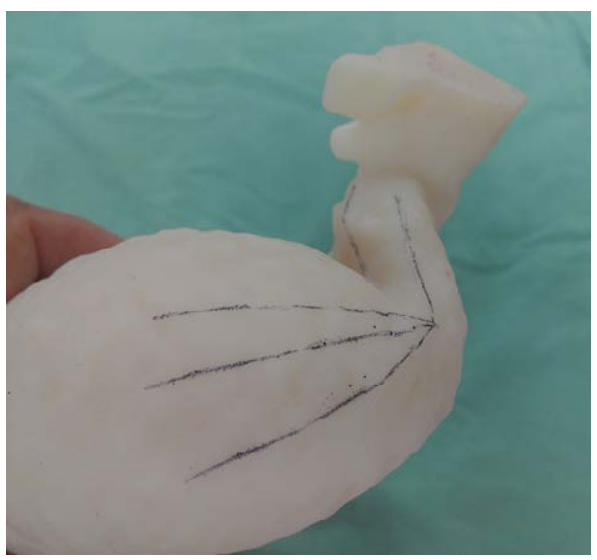

Figure 15: A 3D model of an AAA with severe variation of possible centerlines and angles.

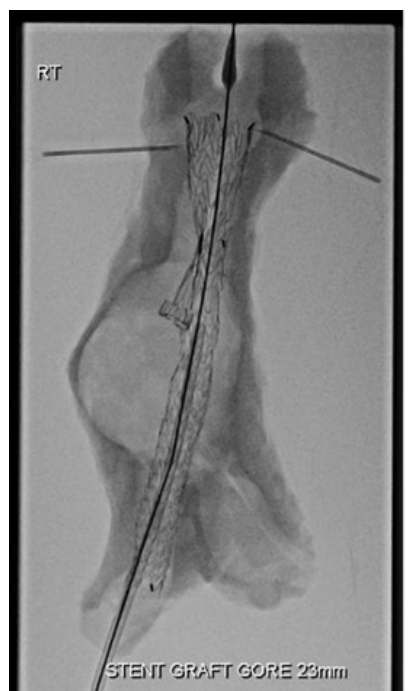

Figure 16: Preoperative simulation of EVAR on an AAA shell. A bifurcated Gore stent graft is completely deployed inside.

neck and a single renal artery it has been suggested by one company representative and a product specialist to be suitable for EVAR with a single covered stent to the renal artery as a "chimney" (Figure 18).

We doubted that EVAR is feasible in this case and performed a preoperative rehearsal simulation with both Gore and Valiant devices. Both devices failed in this simulation. The Gore device seemed to be misaligned to the Aortic wall, and the contra lateral limb seemed to be compressed. The Medtronic device could not take the sharp angulation and it slipped down into the Aneurysmal sac (Figures 19 and 20).

\section{Discussion}

Recent advances in 3D printing technology, in both software and hardware, are making the process of in house preparation of 3D physical models easily accessible in many medical centers. This technology may prove to be very helpful in general surgery and especially in the field of vascular medicine and surgery. Complex AAA disease is a good example, in our opinion, where $3 \mathrm{D}$ technology will become significant in treatment planning and preoperative simulation. Every experienced vascular surgeon and invasive radiologist may want to better see and palpate the minute details of a complex aneurysm that he is going to treat. A rapidly printable $3 \mathrm{D}$ model will be helpful in defining important details like origin of branches, angulations, neck length, stenoses and more. This may determine the choice of optimal techniques and devices. It is expected that $3 \mathrm{D}$ printing will markedly improve results of treatment in these complex AAA patients.

In this study we have demonstrated the feasibility of conquering these technologies by an experienced team of vascular surgeons and invasive radiologists only, without the need of help from engineers and other professionals. All the models presented here were prepared by us and the only other help was a 3D printing machine operator. Since 3D printing services are becoming common worldwide, and less expensive, this means that $3 \mathrm{D}$ printing of AAA will spread shortly and will be used widely. If a $3 \mathrm{D}$ printer is already available in the medical

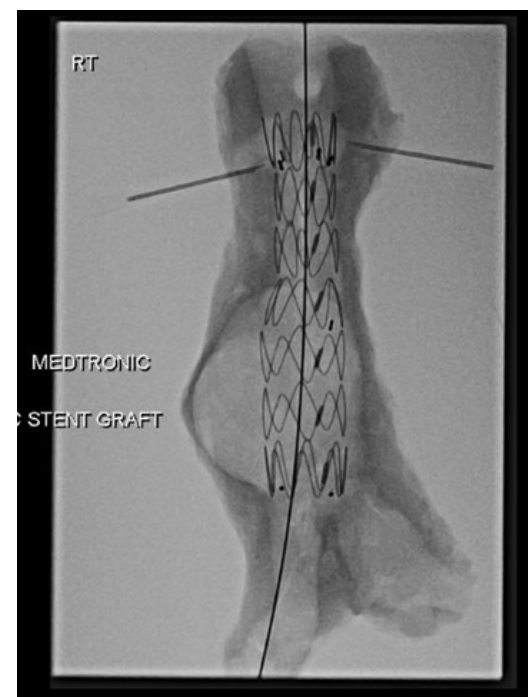

Figure 17: Preoperative simulation of EVAR on an AAA shell. A Medtronic Thoracic tubular stent graft is deployed inside the shell.

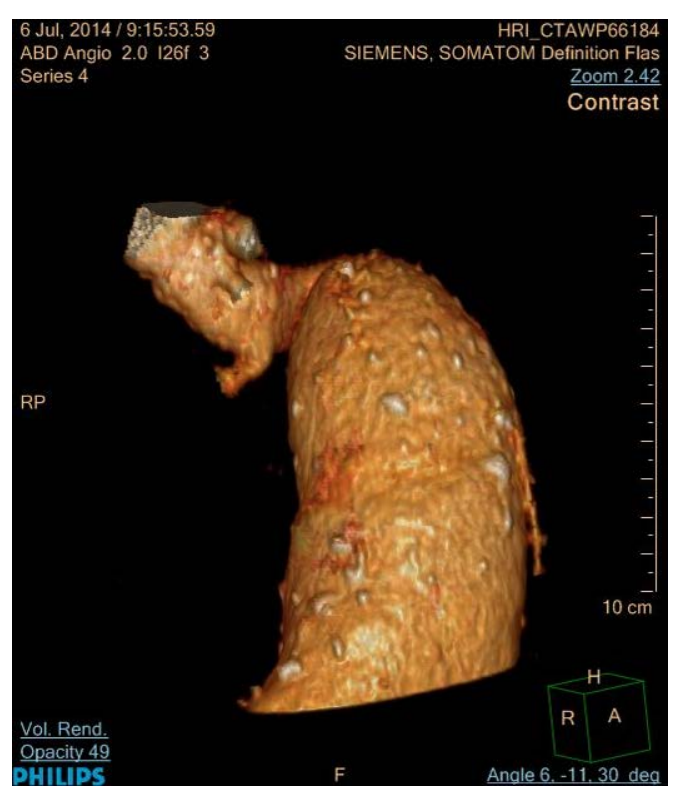

Figure 18: On screen 3D reconstruction of complex AAA neck area. 


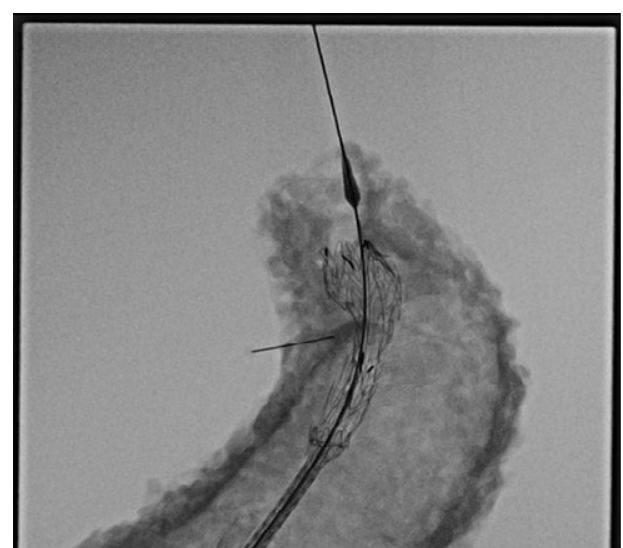

Figure 19: Partially deployed Gore stent graft in a shell of a sharply angulated neck of an AAA. The stent is seen to be misaligned.

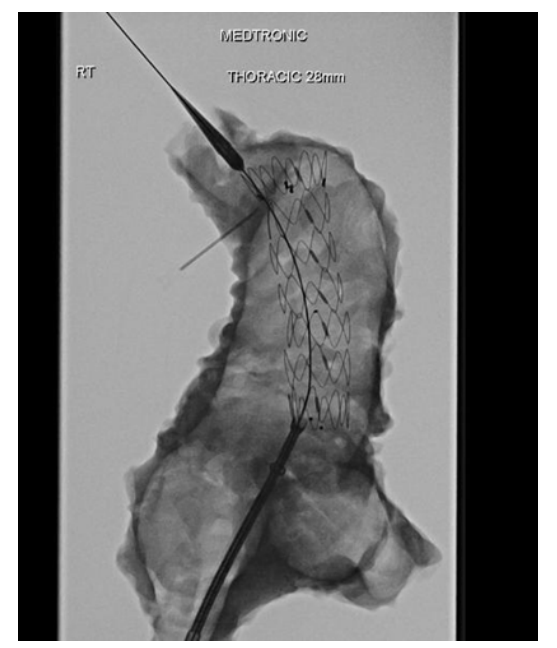

Figure 20: A Medtronic stent graft deployed inside an AAA shell. The device migrated and slipped down into the aneurysm sac.

center then the vascular team itself can prepare the desired models in house, as described in this paper and the more detailed appendices. The vascular team can design the best model according to their previous experience, knowledge and judgment of what is most important to them in the evaluation of a specific area of interest.

In this report containing our preliminary experience with $3 \mathrm{D}$ modeling technology we have demonstated the value of 3D physical models in better comprehension of comlex AAA. However, we would like to emphasize that $3 \mathrm{D}$ physical models are not required for most AAA cases. Computer screen based reconstructions, using the advanced software packages presently available, are sufficient in most cases and provide all technical information needed. However, in a minority of cases, with borderline anatomy, these 3D models, outer shells, and preopertive simulation, may be helpful for the decision making of feasibility of EVAR and for the techniques that will enable it safely.

In the future we hope to see the technology used to build personally fitted stent and stent-grafts, with branches and fenestrations fabricated precisely for the specific complex patient's unique anatomy, as part of the trend towards personalized medicine. These advances will be of help in diagnosis and treatment planning of complex anatomical and pathological conditions not only in vascular surgery but in many other areas in medicine and surgery.

\section{Acknowledgements}

We would like to thank Itamar Kahn for collaboration in this project, and Guennady Yudkovsky for excellent assistance in printing the 3D models.

\section{References}

1. Parker MV, O'Donnell SD, Chang AS, Johnson CA, Gillespie DL, et al. (2005) What imaging studies are necessary for abdominal aortic endograft sizing? A prospective blinded study using conventional computed tomography, aortography, and three-dimensional computed tomography. J Vasc Surg 41: 199-205.

2. Kim MS, Hansgen AR, Wink O, Quaife RA, Carroll JD (2008) Rapid prototyping: a new tool in understanding and treating structural heart disease. Circulation 117: $2388-2394$

3. Knox K, Kerber CW, Singel SA, Bailey MJ, Imbesi SG (2005) Rapid prototyping to create vascular replicas from CT scan data: making tools to teach, rehearse, and choose treatment strategies. Catheter Cardiovasc Interv 65: 47-53.

4. Lermusiaux P, Leroux C, Tasse JC, Castellani L, Martinez R (2001) Aortic aneurysm: construction of a life-size model by rapid prototyping. Ann Vasc Surg 15: 131-135.

5. Berry E, Marsden A, Dalgarno KW, Kessel D, Scott DJ (2002) Flexible tubular replicas of abdominal aortic aneurysms. Proc Inst Mech Eng H 216: 211-214. 